\title{
Intervention for Non-verbal and Minimally-Verbal Individuals with Autism: A Systematic Review
}

\author{
Lynn Kern Koegel, PhD ${ }^{1 *}$, Katherine M Bryan, BA ${ }^{2}$, Pumpki Su, MA², Mohini Vaidya ${ }^{3}$ and Stephen \\ Camarata, PhD (iD $^{2}$ \\ ${ }^{1}$ Department of Psychiatry and Behavioral Sciences, Stanford School of Medicine, Stanford University, USA \\ ${ }^{2}$ School of Medicine, Vanderbilt University, USA \\ ${ }^{3}$ University of California, Santa Barbara, USA

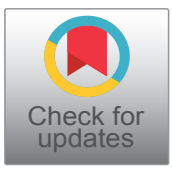

*Corresponding author: Lynn Kern Koegel, Department of Psychiatry and Behavioral Sciences, Stanford School of Medicine, Stanford University, Palo Alto, CA 94305, USA

\begin{abstract}
Background: Most children diagnosed with ASD will present with late onset verbal communication, and at least one third of these children will remain minimally or completely nonverbal throughout their lifespan, speaking few or no words. Challenges with verbal language can negatively affect many areas, including socialization, academics, independent living and employment. The objective of this paper was to systematically review interventions for the treatment of nonverbal and minimally verbal individuals with ASD. This review exclusively selected studies that targeted verbal communication in minimally and nonverbal individuals diagnosed with ASD. The interventions provided, the outcomes of these interventions, measures used to assess change, and pre- post measures were included.
\end{abstract}

Question: What is the current evidence base for interventions for non- and minimally verbal individuals with Autism Spectrum Disorder (ASD)?

Methods: A literature search was conducted through ProQuest (Mendeley reference manager). Articles were extrapolated from seventy data bases. Databases surveyed included Medline, Periodicals Archive Online, Periodicals Index Online, PRISMA Database, ProQuest Central, PsycARTICLES and PsycINFO. A complete list of all databases included is available on ProQuest (http://tls.search.proquest. com/titlelist/jsp/list/tlsSingle.jsp?productld=10000255).

The publication span entered was 1960 to 2018 . Reliability for coding was examined and was uniformly above $90 \%$ concordance.

Results: Our search yielded 2,007 articles, of which 29 studies met our inclusion criteria. Inclusion criteria included: (1) Research Design: Studies that involved systematic, experimentally controlled investigations, such as randomized controlled trials, quasi-experimental designs, and single-case designs; (2) Diagnosis: only studies that included minimally verbal, nonverbal, and preverbal participants diagnosed with ASD were included; and (3) Targeted verbal communication: the goal of the intervention was to initiate or improve verbal communication, including the production of words, word attempts, or sounds. This review found that there was a wide variety of interventions provided, comparisons across interventions were lacking, and dependent measures varied considerably.

Conclusions: Presently, pediatricians and service providers are unable to provide evidence-based treatment recommendations regarding speech and language interventions for non- and minimally verbal individuals with ASD. Limited evidence suggests that verbal-focused treatments in natural settings with parent participation is effective, as research regarding the most effective and efficient interventions for this high need group is lacking. Lack of uniformity in regard to dependent and pre- post measures, participant ages, and description of interventions implemented make comparing outcomes across studies difficult. Uniform standards for identifying MV and NV children with ASD is needed in future studies and additional details on the intervention procedures in futures studies is also needed.

\section{Keywords}

Autism, Nonverbal, Minimally verbal, Expressive words, Communication treatment

\section{Abbreviations}

ASD: Autism Spectrum Disorder; MV: Minimally-Verbal; NV: Non-Verbal

Citation: Koegel LK, Bryan KM, Su P, Vaidya M, Camarata S (2019) Intervention for Non-verbal and Minimally-Verbal Individuals with Autism: A Systematic Review. Int J Pediatr Res 5:056. doi. org/10.23937/2469-5769/1510056

Accepted: September 25, 2019: Published: September 27, 2019

Copyright: (c) 2019 Koegel LK, et al. This is an open-access article distributed under the terms of the Creative Commons Attribution License, which permits unrestricted use, distribution, and reproduction in any medium, provided the original author and source are credited. 


\section{Key Points}

\section{Findings}

In this systematic review, no consistencies across intervention recommendations were identified. For example, some studies recommended the use of augmentative programs (signs/pictures/computer programs) along with or before targeting verbal communication whereas other studies indicated that augmentative programs are not necessary. Regardless of program, most recommend the use of naturalistic intervention procedures.

\section{Meaning}

Currently, there are no consistent recommendations for pediatricians and practitioners to make for treating nonverbal and minimally verbal children with ASD. Systematic intervention research for this population is needed.

\section{Introduction}

Parents rely on pediatricians and service providers for referral to effective interventions when their child is diagnosed ASD. Although social and behavioral symptoms of ASD may manifest in the first year of life, the delayed onset of language is the most common reason for a diagnostic consultation by parents [1] and is one of the most concerning symptoms of ASD [2]. Although many children with ASD are "high functioning" and communicate verbally, a high proportion will persist as nonverbal or minimally verbal.

Recently, the literature has highlighted the pressing need to study children with ASD who have extremely limited verbal abilities, identifying this subgroup of ASD as grossly under-represented in the intervention literature [3-5]. That is, in the last almost 60 years, relatively few studies have focused on intervention for targeting first word production in this population after the age of two years [6]. In the absence of specific guidelines and definitive strategies supported by objective data, children with ASD may receive ineffective or misguided interventions. Training programs, clinics, and schools cannot deliver best practices, and children with ASD may suffer the consequences of an inability to verbally communicate in the absence of a credible evidence base. To be clear, nearly all children with ASD are nonverbal or low verbal as toddlers, but many of these children become verbal before age three years. The focus of this review is on children with ASD who persist as nonverbal or low verbal after two years of age.

\section{Pathophysiology of MV and NV ASD}

According to the DSM-5 [7], a defining characteristic of ASD is persistent deficits in social communication. Typically developing children produce first words between 10 and 18 months, whereas children with ASD are reported to do so at an average age of 36 months [8]. Further, a third of children diagnosed with ASD will remain minimally verbal or totally nonverbal [9].

\section{Clinical presentation of MV and NV ASD}

The late onset of communication in children who will be subsequently diagnosed with ASD is the primary reason parents bring their child in for initial evaluation [1]. Challenges with social communication along with restricted and repetitive behaviors (RRBs) are required for the diagnosis of ASD. RRBs often present as repetitive play in young children and few interests in older individuals. Challenges with socialization, particularly when interacting with peers, tend to persist across the lifespan, even for those who develop age appropriate communication. ASD encompasses a largely heterogeneous group, and symptomology varies considerably across individuals ranging from nonverbal/minimally verbal to highly verbal. RRBs range from repetitive motor behaviors to excessive interest in particular, often idiosyncratic topics. Those children with ASD falling into the persistent NV and MV subtype also have a higher probability of generally slower intellectual development and a higher incidence of behavioral sequelae.

This systematic review analyzes current intervention studies for this population, outcomes, and measures used to assess change. We reviewed studies since 1960 when interventions for ASD were first published. Outcomes are analyzed and recommendations are provided.

\section{Methods}

A literature search with a publication span from 1960 to 2018 was executed using Vanderbilt's ProQuest using Mendeley reference manager (https://www.mendeley. com/reference-management/reference-manager) from seventy data bases using the key words "autism", "autistic", "Asperger", "autisms", or "ASD" AND "minimally verbal", "minimally fluent", "preverbal", "pre-verbal", "nonverbal", or "non-verbal" "mute" AND "vocabulary", "words", "communication", "language", or "lexical." ProQuest databases surveyed included Medline, Periodicals Archive Online, Periodicals Index Online, PRISMA Database, ProQuest Central, PsycARTICLES and PsycINFO. A complete list of all databases included is available at ProQuest (http://tls.search.proquest.com/titlelist/jsp/list/ tlsSingle.jsp?productld=10000255). This search yielded a total of 2007 articles. Only articles with interventions provided in English were included because the authors were not qualified to review interventions in other languages. Duplicates were removed, and a title screening to exclude articles that did not include nonverbal or minimally verbal individuals or did not provide treatment yielded 237 articles with $90 \%$ reliability for inter-reviewer article triage.

\section{Inclusion and Exclusion Criteria}

Only scholarly articles and peer-reviewed articles were included. Reports, dissertations, conference papers, and/or proceedings were not included. Additionally, articles were excluded if the titles indicated that they (a) Were assessment only or other non-treatment articles; (b) Did not target verbal be- 
havior(s) (e.g., receptive communication, reading, vision); (c) Included highly verbal participants or advanced communication goals (e.g., Asperger Disorder, conversation, language structures); (d) Were commentaries, book reviews, reviews of the literature, errata; (e) Had fewer than two participants; or (f) Were conducted in a spoken language other than English. The final criterion was adopted due to the authors' and coders' limited expertise in the pantheon of spoken languages appearing in the topic search rather than any prior assumption as to the relative advantages or disadvantages of any particular spoken language.

\section{Title screening}

First, all of the titles were independently read and screened by the first (primary coder) and last (reliability coder) authors, who had the most experience in the field, using the following inclusion criteria:

1. Titles that included "minimally verbal" children with ASD

2. Titles that included "nonverbal" children with ASD

3. Treatment/intervention articles targeting verbal communication skills

Duplicates were removed, yielding a total of 1,231 articles. This title search yielded 237 articles out of 1,231. Reliability for the title screen of the articles was $90 \%$. Articles from the title screening that were included by only one coder (either the primary or reliability coder) were included for the abstract screening.

\section{Abstract screening}

Following the title search, abstracts from the 237 articles were screened using the additional following specific inclusion and exclusion criteria:

Research design: Studies that involved systematic, experimentally controlled investigation intervention were included. Example research designs meeting inclusion criteria were randomized controlled trials, quasi-experimental designs, and single-case designs with at least two participants. Uncontrolled case studies (e.g., $\mathrm{N}=1$ ) were excluded from this review. In order to ensure the study was evaluating intervention effectiveness, at least one dependent variable had to be a child outcome measure.

Treatment: Only articles that implemented an intervention for (or that resulted in) expressive verbal communication or interventions to evoke first words as the independent variable were included.

Diagnosis: Participants, or the majority of participants in the study, were required to have been diagnosed with ASD, and the participants diagnosed with ASD and had to have been identified as minimally verbal, nonverbal, preverbal or another description of the communication abilities of the participants relating to the production of first words.

Measurement: Included studies involved verbal behavior (words, word attempts, or sounds) as a dependent variable. Nonverbal modes of communication-augmentative, sign language, etc.-that did not include verbal output or explicitly state that the approach was used as a bridge to verbal communication were excluded. That is, articles that included picture supports, or other visual symbols were included only if the outcome dependent measure(s) were focused on verbal communication. Similarly, voice generating devices (e.g., touch talkers, DynaVox systems) were excluded unless the outcome measure(s) focused on verbal communication by the child.

Participants: Individuals diagnosed with Asperger Disorder, PDD-NOS, or whose target behavior included socialization, receptive language, non-verbal communication exclusively, or whose treatment program included an augmentative system or nonverbal communicative system (e.g., pointing) that were not explicitly a precursor to verbal speech were also excluded.

The first (primary coder) and last (reliability coder) authors screened the first 50 articles with $96 \%$ reliability. The second and third authors screened the first 50 abstracts with $87 \%$ reliability. Next, half of the abstracts from the 237 included from the title review were screened by the first (primary coder) and fourth (reliability coder) author and the remainder were screened by the second (primary coder) and third (reliability coder) author. The reliability for the abstract selection was $89 \%$. Any abstracts that were included by only one coder (either the primary or reliability coder) were included for the full article review. The abstract search yielded 67 articles.

These 67 articles that specified intervention to individuals who were nonverbal or minimally verbal were read in full and analyzed for a parent education program. Nineteen of the 67 articles (28\%) were reviewed for reliability purposes relating to inclusion/ exclusion. During the write-up, the first author found two articles that appeared to meet our exclusion criteria. The last author read these articles and concurred that they should be excluded from the analysis (one was conducted in a foreign language, and the other targeted nonverbal social behavior in individuals with Asperger Disorder who were highly verbal). Thus, reliability on inclusion/exclusion was $89 \%$.

The 29 articles meeting the inclusion criteria were then fully reviewed and coded for: (a) Age of participants/sample size/male-female inclusion rates; (b) Dependent measures; (d) Pre- post intervention measures; (d) Descriptions of treatment provided; and (e) Study outcome. Following the creation of the summary table (Table 1), an independent coder re- 
Table 1: Treatments used in the studies for nonverbal and minimally-verbal children with ASD.

\begin{tabular}{|c|c|c|c|c|c|}
\hline $\begin{array}{l}\text { Author \& } \\
\text { Year }\end{array}$ & $\begin{array}{l}\mathbf{N}(\mathrm{m} / \mathrm{f}) \\
\text { Age }\end{array}$ & $\begin{array}{l}\text { Dependent Mea- } \\
\text { sures }\end{array}$ & Pre-post Measures & Treatment & Outcome \\
\hline $\begin{array}{l}\text { Almirall D, } \\
\text { et al. [10] }\end{array}$ & $\begin{array}{l}61(51 \\
10)\end{array}$ & $\begin{array}{l}\text { Five DVs: } \\
\text { \# Total sponta- } \\
\text { neous communi- } \\
\text { cative utterances } \\
\text { from naturalistic } \\
\text { language sample } \\
\text { \# Number of differ- } \\
\text { ent word root } \\
\text { \# Initiating joint at- } \\
\text { tention (IJA) and \# } \\
\text { initiating behavior- } \\
\text { al regulation (IBR) } \\
\text { from ESCS } \\
\text { \# Total number of } \\
\text { unique play from } \\
\text { structure play } \\
\\
\text { Expressive: Total } \\
\text { number of sponta- } \\
\text { neous communi- } \\
\text { cative utterances } \\
\text { (TSCU), number } \\
\text { of different word } \\
\text { roots (NDWR), }\end{array}$ & $\begin{array}{l}\text { \# Leiter - R } \\
\text { \# ADOS } \\
\text { \# 20-min naturalistic } \\
\text { language sample } \\
\text { (NLS): Total number } \\
\text { of spontaneous com- } \\
\text { municative utterances } \\
\text { (TSCU), \% sponta- } \\
\text { neous communicative } \\
\text { utterances, number } \\
\text { of different word } \\
\text { roots, MLU, word per } \\
\text { minute (WPM), total } \\
\text { number of comments } \\
\text { (TCOM), unique word } \\
\text { combination (UWC), } \\
\text { and total number of } \\
\text { spontaneous requests } \\
\text { (SPRQ) } \\
\text { \# ESCS: IJA and IBR } \\
\text { \# Structure Play } \\
\text { Assessment: Total } \\
\text { number of unique play } \\
\text { action (UPA) }\end{array}$ & $\begin{array}{l}\text { SMART design that } \\
\text { includes two stages } \\
\text { of tx. The first stage } \\
\text { assigned children to } \\
\text { JASP + EMT or JASP } \\
+ \text { EMT + SGD, second } \\
\text { stage quick respond- } \\
\text { ers and slow respond- } \\
\text { ers were identified. } \\
\text { Quick responders stay } \\
\text { in the same treatment } \\
\text { while slow responders } \\
\text { were assigned to ei- } \\
\text { ther intensified JAST } \\
+ \text { EMT or augmented } \\
\text { JASP + EMT + SGD. }\end{array}$ & $\begin{array}{l}\text { Sig differences in slopes (at } \\
\text { Stage } 1 \text { or Stage 2) among the } \\
\text { three adaptive interventions on } \\
\text { two of the five outcomes consid- } \\
\text { ered: TSCU ( } p<0.01 \text { ) and IJA ( } p \\
=0.046) \\
\text { Authors concluded among the } \\
\text { three adaptive interventions, } \\
\text { the (SGD, SGD) adaptive in- } \\
\text { tervention was found to lead to } \\
\text { improved spontaneous spoken } \\
\text { communicative utterances and } \\
\text { initiating joint attention relative } \\
\text { to (No SGD, No SGD) and (No } \\
\text { SGD, SGD). }\end{array}$ \\
\hline $\begin{array}{l}\text { Chenausky } \\
\text { K, et al. } \\
{[30]}\end{array}$ & $\begin{array}{l}30(27, \\
3) \\
3 ; 5-9 ; 8\end{array}$ & $\begin{array}{l}\text { \% Syllables Ap- } \\
\text { proximated } \\
\% \text { Consonants } \\
\text { Correct } \\
\% \text { Vowels Correct }\end{array}$ & $\begin{array}{l}\text { Probe assessments } \\
\text { of repeating } 15 \text { high } \\
\text { frequency ( } 30 \text { total) } \\
\text { bisyllabic words or } \\
\text { phrases, trained and } \\
\text { untrained. }\end{array}$ & $\begin{array}{l}\text { Auditory Motor } \\
\text { Mapping Training } \\
\text { (Listening/unison, } \\
\text { unison fade, imitate, } \\
\text { and cloze) compared } \\
\text { with AMMT + Speech } \\
\text { Repetition Treatment } \\
\text { (SRT) }\end{array}$ & $\begin{array}{l}\text { After } 25 \text { sessions, AMMT par- } \\
\text { ticipants increased by } 19.4 \% \\
\text { syllables approximated, } 13.8 \% \\
\text { consonants correct, and } 19.1 \% \\
\text { vowels Correct, compared to } \\
\text { best baseline. In the matched } \\
\text { AMMT-SRT group, after } 25 \\
\text { sessions, AMMT participants } \\
\text { produced } 29.0 \% \text { more syllables } \\
\text { approximated (SRT 3.6\%); } 17.9 \% \\
\text { more consonants correct (SRT } \\
\text { 0.5); and } 17.6 \% \text { more vowels } \\
\text { correct (SRT 0.8\%). }\end{array}$ \\
\hline $\begin{array}{l}\text { DiStefano, } \\
\text { et al. [11] }\end{array}$ & $\begin{array}{l}55 \text { (Not } \\
\text { report- } \\
\text { ed) }\end{array}$ & $\begin{array}{l}\text { Total number of } \\
\text { different words, } \\
\text { total spontaneous } \\
\text { communicative } \\
\text { utterances, total \# } \\
\text { of comments ("as } \\
\text { well as other vari- } \\
\text { ables) }\end{array}$ & $\begin{array}{l}10 \text { min samples at en- } \\
\text { try and } 1 x \text { per month } \\
\text { of intervention }\end{array}$ & $\begin{array}{l}\text { Half received play and } \\
\text { engagement interven- } \\
\text { tion that incorporated } \\
\text { SGD for } 6 \text { months; } \\
45-60 \text { mins } 2 x / \text { week } \\
\text { months } 1-3 \text {. Slow re- } \\
\text { sponders switched to } \\
\text { increased intensity or } \\
\text { original condition }\end{array}$ & $\begin{array}{l}\text { Those higher at BL did better } \\
\text { JASPER-EMT + SGD did slightly } \\
\text { better } \\
\text { Children in both groups improved } \\
\text { in long interchanges; site differ- } \\
\text { ences in short interchanges } \\
\text { Entry \# of interchanges correlat- } \\
\text { ed with exit } \\
\text { TDWR (words at entry correlated } \\
\text { with exit) } \\
\text { Spontaneous (TSCU) utterances } \\
\text { at entry not predictor of TDWR } \\
\text { at exit however entry \# of inter- } \\
\text { changes and TSCU significant } \\
\text { indicator of exit TSCU not mea- } \\
\text { sured } \\
\text { by spontaneous language sam- } \\
\text { ple only during intervention }\end{array}$ \\
\hline
\end{tabular}




\begin{tabular}{|c|c|c|c|c|c|}
\hline $\begin{array}{l}\text { Drash PW, } \\
\text { et al. [14] }\end{array}$ & $\begin{array}{l}3(3,0) \\
2 ; 6-3 ; 6\end{array}$ & $\begin{array}{l}\% \text { of mands (any } \\
\text { vocal response to } \\
\text { prompt, excluding } \\
\text { inappropriate vo- } \\
\text { cal behavior); \% } \\
\text { of correct echoic } \\
\text { responses; \% of } \\
\text { error responses; } \\
\% \text { of no responses } \\
\text { and inappropriate } \\
\text { behavior com- } \\
\text { bined; \% of tact } \\
\text { responses }\end{array}$ & $\begin{array}{l}\% \text { of mands (any vocal } \\
\text { response to prompt, } \\
\text { excluding inappropri- } \\
\text { ate vocal behavior); } \\
\% \text { of correct echoic } \\
\text { responses; \% of error } \\
\text { responses; \% of no } \\
\text { responses and inap- } \\
\text { propriate behavior } \\
\text { combined; \% of tact } \\
\text { responses }\end{array}$ & $\begin{array}{l}\text { Shaping the mand rep- } \\
\text { ertoire by using estab- } \\
\text { lishing operations and } \\
\text { specific reinforcers. }\end{array}$ & $\begin{array}{l}\text { All } 3 \text { children acquired an initial } \\
\text { echoic repertoire within the } 1^{\text {st }} \\
10 \text { sessions. Two of the children } \\
\text { also began tacting; all partici- } \\
\text { pants acquired a mand repertoire } \\
\text { by the } 6^{\text {th }} \text { session; negative vocal } \\
\text { behavior decreased rapidly }\end{array}$ \\
\hline $\begin{array}{l}\text { Esch JW, } \\
\text { et al. [31] }\end{array}$ & $\begin{array}{l}2(2,0) \\
2 ; 6 \& \\
7 ; 1\end{array}$ & $\begin{array}{l}\text { \# Frequency of } \\
\text { varied vocaliza- } \\
\text { tion: Varied vocal } \\
\text { response defined } \\
\text { as a speech vo- } \\
\text { calization that was } \\
\text { different from the } \\
\text { response of the } \\
\text { preceding trial } \\
\text { and that occurred } \\
\text { within } 5 \text { s of the ex- } \\
\text { perimenter's model } \\
\text { (coded as D) }\end{array}$ & $\begin{array}{l}\text { Pre-intervention as- } \\
\text { sessment include a } \\
\text { phoneme imitation } \\
\text { task, play sample, and } \\
\text { Kaufman speech Prax- } \\
\text { is test for children }\end{array}$ & $\begin{array}{l}\text { Discrete Trial Training: } \\
\text { Reinforcer delivered } \\
\text { when a response is } \\
\text { different from the re- } \\
\text { sponse immediately } \\
\text { preceding it }\end{array}$ & $\begin{array}{l}\text { Authors concluded "systematic } \\
\text { increases in varied vocalization" } \\
\text { in both children but because of } \\
\text { the large variability }\end{array}$ \\
\hline $\begin{array}{l}\text { Franco JH, } \\
\text { et al. [22] }\end{array}$ & $\begin{array}{l}6(5,1) \\
5 ; 1-8 ; 3\end{array}$ & $\begin{array}{l}\text { \# the number } \\
\text { of acts that the } \\
\text { children used to } \\
\text { maintain social } \\
\text { interaction during } \\
\text { each routine } \\
\text { \# rate/minute } \\
\text { of child initiated } \\
\text { intentional com- } \\
\text { munication (total } \\
\text { number of child-ini- } \\
\text { tiated communica- } \\
\text { tion acts divided } \\
\text { by total number } \\
\text { of minutes in the } \\
\text { session) } \\
\text { HYBRID: Included } \\
\text { vocalizations into a } \\
\text { larger communica- } \\
\text { tion variable. The } \\
\text { reported measure } \\
\text { was a combination } \\
\text { of verbal and non- } \\
\text { verbal }\end{array}$ & $\begin{array}{l}\text { Single subject design } \\
\text { No pre-post tests } \\
\text { Baseline was omnibus } \\
\text { communication vari- } \\
\text { able }\end{array}$ & $\begin{array}{l}\text { Prelinguistic Milieu } \\
\text { Teaching using } \\
\text { techniques including } \\
\text { prompts, models, and } \\
\text { natural consequences } \\
\text { in a naturalistic play } \\
\text { context }\end{array}$ & $\begin{array}{l}\text { participants did not demonstrate } \\
\text { intentional communication at } \\
\text { baseline. During intervention, } \\
\text { all six children increased in the } \\
\text { rates of initiation of intentional } \\
\text { communication compared to } \\
\text { baseline. } \\
\text { Improvement rate difference } \\
\text { (IRD) was used as effect size: } \\
\% 90 \text { for acts to maintain social } \\
\text { interaction; \%87 for rate/minute } \\
\text { of child initiated intentional com- } \\
\text { munication. Pooled vocalizations } \\
\text { with nonverbal social communi- } \\
\text { cation measures }\end{array}$ \\
\hline
\end{tabular}




\begin{tabular}{|c|c|c|c|c|c|}
\hline $\begin{array}{l}\text { Green J, et } \\
\text { al. [15] }\end{array}$ & $\begin{array}{l}152 \\
(124, \\
28) \\
2 ; 0-4 ; 11\end{array}$ & $\begin{array}{l}\text { Primary: Severity } \\
\text { of the symptoms of } \\
\text { autism } \\
\text { Secondary: (1) } \\
\text { Parent-child in- } \\
\text { teraction during } \\
\text { naturalistic play } \\
\text { in a non-therapy } \\
\text { setting (2) Child } \\
\text { language and so- } \\
\text { cial communication } \\
\text { (3) Adaptive func- } \\
\text { tioning in school } \\
\text { beyond the family. } \\
\\
\text { Hybrid: ADOS } \\
\text { social communica- } \\
\text { tion does include } \\
\text { verbal words and } \\
\text { verbal communi- } \\
\text { cation. But, the } \\
\text { growth in verbal } \\
\text { skills could not be } \\
\text { ascertained. }\end{array}$ & $\begin{array}{l}\text { Primary measure: } \\
\text { ADOS-G social com- } \\
\text { munication algorithm } \\
\text { score (scoring pro- } \\
\text { cedure modified to } \\
\text { improve sensitivity to } \\
\text { change) } \\
\text { Secondary measures: } \\
\text { (1) Video tapes from } \\
\text { the study were as- } \\
\text { sessed for proportion } \\
\text { of parental communi- } \\
\text { cations with the child } \\
\text { that were synchro- } \\
\text { nous, proportion of } \\
\text { child communications } \\
\text { with the parent that } \\
\text { were initiations, and } \\
\text { proportion of time } \\
\text { spent in mutual } \\
\text { shared attention (2) } \\
\text { Assessed by the re- } \\
\text { searcher using the } \\
\text { Preschool Language } \\
\text { Scales and reported } \\
\text { by the parent accord- } \\
\text { ing to the MacArthur } \\
\text { Communicative De- } \\
\text { velopment Inventory } \\
\text { (MCDI, infant form raw } \\
\text { scores) and the Com- } \\
\text { munication and Sym- } \\
\text { bolic Behavior Scales } \\
\text { Developmental Profile } \\
\text { (CSBS-DP, caregiver } \\
\text { questionnaire) social } \\
\text { composite raw scores } \\
\text { (3) Assessed by Vine- } \\
\text { land Adaptive Behav- } \\
\text { ior Scales (VABS), } \\
\text { Teacher Rating Form, } \\
\text { and rated at endpoint } \\
\text { by face-to-face inter- } \\
\text { view with teachers in } \\
\text { nurseries, reception } \\
\text { class, or other appro- } \\
\text { priate caregiver who } \\
\text { was not a member of } \\
\text { the family }\end{array}$ & $\begin{array}{l}\text { Preschool Autism } \\
\text { Communication Trial } \\
\text { (PACT) } \\
\text { parent-mediated com- } \\
\text { munication-focused } \\
\text { intervention }\end{array}$ & $\begin{array}{l}\text { Primary: No significant improve- } \\
\text { ment in severity of autism symp- } \\
\text { toms in comparison to control } \\
\text { group } \\
\text { Secondary: (1) Intervention } \\
\text { effects were strong for asses- } \\
\text { sor-rated parent-child inter- } \\
\text { actions (parent synchronous } \\
\text { responses and child communica- } \\
\text { tion initiations, although less for } \\
\text { shared attention). (2) No signifi- } \\
\text { cant effect on directly assessed } \\
\text { language (3) No significant effect } \\
\text { on adaptive functioning in school. } \\
\text { Unknown whether there were } \\
\text { changes in vocalizations/verbal } \\
\text { skills (not reported) }\end{array}$ \\
\hline $\begin{array}{l}\text { Gevarter C, } \\
\text { et al. [32] }\end{array}$ & $\begin{array}{l}6(5,1) \\
3 ; 6-5 ; 3\end{array}$ & $\begin{array}{l}\text { Independent and } \\
\text { prompted vocaliza- } \\
\text { tions, approxima- } \\
\text { tion of target word, } \\
\text { full words }\end{array}$ & $\begin{array}{l}\text { No pre-post tests } \\
\text { single subject design }\end{array}$ & $\begin{array}{l}\text { Whole words targeted. } \\
\text { Any vocalization was } \\
\text { rewarded. Rewards for } \\
\text { DT for word production }\end{array}$ & $\begin{array}{l}5 / 6 \text { showed increased vocaliza- } \\
\text { tions }\end{array}$ \\
\hline
\end{tabular}




\begin{tabular}{|c|c|c|c|c|c|}
\hline $\begin{array}{l}\text { Gordon K, } \\
\text { et al. [33] }\end{array}$ & $\begin{array}{l}84(73, \\
11) \\
4-10\end{array}$ & $\begin{array}{l}\text { initiations, request- } \\
\text { ing, use of PECS } \\
\text { picture cards } \\
\text { Hybrid: Included } \\
\text { nonverbal and } \\
\text { verbal initiations. } \\
\text { Vocalizations as a } \\
\text { separate measure. } \\
\text { (Spontaneous } \\
\text { initiation using } \\
\text { speech/vocaliza- } \\
\text { tion (IC-S) }\end{array}$ & $\begin{array}{l}\text { The primary outcome } \\
\text { variable was frequen- } \\
\text { cy of child-initiated } \\
\text { communication (IC). } \\
\text { Frequencies of differ- } \\
\text { ent communication } \\
\text { modalities used (such } \\
\text { as the number of } \\
\text { times a child used a } \\
\text { picture card (P) and/ } \\
\text { or speech/vocalization } \\
\text { (S) to communicate) } \\
\text { were also recorded; } \\
\text { communication func- } \\
\text { tions were recorded } \\
\text { by counting each time } \\
\text { a child communicated } \\
\text { for the purpose of re- } \\
\text { questing objects (R) } \\
\text { and for the purpose of } \\
\text { requesting a social in- } \\
\text { teraction or comment- } \\
\text { ing (D). In this way, a } \\
\text { single communication } \\
\text { act might produce } 3 \text { or } \\
\text { more codes, e.g. as a } \\
\text { spontaneous initiation } \\
\text { (IC), of the use of a } \\
\text { picture card (P) and } \\
\text { for the purpose of re- } \\
\text { questing (R). }\end{array}$ & $\begin{array}{l}\text { PECS phase } 1 \text { with cli- } \\
\text { nician verbal modeling } \\
\text { of words }\end{array}$ & $\begin{array}{l}\text { There were positive moderated } \\
\text { treatment effects } \\
\text { Requesting (mands) for objects. } \\
\text { Social communication did not } \\
\text { improve } \\
21 \text { participants (those who were } \\
\text { more severe at baseline) showed } \\
\text { no gains }\end{array}$ \\
\hline $\begin{array}{l}\text { Harris SL, } \\
\text { et al. [16] }\end{array}$ & $\begin{array}{l}11(10 \\
1) \\
2 ; 3-4 ; 6\end{array}$ & $\begin{array}{l}\text { Two half hour Vid- } \\
\text { eotapes ( } 1 \text { week } \\
\text { apart) scored for } \\
\text { Speech/speech at- } \\
\text { tempts, other, and } \\
\text { non-intelligible }\end{array}$ & $\begin{array}{l}\text { 10-item test of facts } \\
\text { about teaching } \\
\text { speech, a consumer } \\
\text { satisfaction question- } \\
\text { naire (Likert scale), } \\
\text { clinical data for be- } \\
\text { havior modification or } \\
\text { speech training }\end{array}$ & $\begin{array}{l}\text { Treatment behavior } \\
\text { modification } \\
\text { Brief summary of pre- } \\
\text { vious week, 40-min } \\
\text { lecture, demonstra- } \\
\text { tions, feedback } 5 \text { mins } \\
\text { discussing individual } \\
\text { academic or behavior } \\
\text { progs } \\
\text { Reading material } \\
\text { Speech - behavior, } \\
\text { nonverbal imitation, } \\
\text { shaping sounds, } \\
\text { teaching nouns, teach- } \\
\text { ing adjectives \& Verbs, } \\
\text { generalization } \\
\text { Weekly group meet- } \\
\text { ings; Home visits ev- } \\
\text { ery } 2 \text { weeks }\end{array}$ & $\begin{array}{l}\text { Pre-post analyses MBL design } \\
\text { with two matched groups } \\
\text { Significant increase in knowledge } \\
\text { after both trainings. } \\
\text { Improvements in speech-ori- } \\
\text { ented language of parents after } \\
\text { speech training. } \\
\text { Verbal children at pre showed } \\
\text { greater improvement than non- } \\
\text { verbal children at post }\end{array}$ \\
\hline
\end{tabular}




\begin{tabular}{|c|c|c|c|c|c|}
\hline $\begin{array}{l}\text { Hingtgen } \\
\text { JN, et al. } \\
{[34]}\end{array}$ & $\begin{array}{l}4(4,0) \\
4 ; 0-5 ; 11\end{array}$ & $\begin{array}{l}\text { \# Number of imi- } \\
\text { tated use-of-body } \\
\text { response } \\
\text { \# Number of imi- } \\
\text { tated use-of-object } \\
\text { response } \\
\text { \# Number of imi- } \\
\text { tated sounds and } \\
\text { words. }\end{array}$ & No pre-post tests & $\begin{array}{l}\text { Three to five weeks } \\
\text { of intensive imitative } \\
\text { training, } \\
\text { consisting of five to six } \\
\text { hours of daily training } \\
\text { sessions, where adults } \\
\text { used reinforcers to } \\
\text { shape imitative behav- } \\
\text { iors: Body response, } \\
\text { use of objects, and re- } \\
\text { ceptive and expressive } \\
\text { language (following } \\
\text { directions, item identifi- } \\
\text { cation, naming items) }\end{array}$ & $\begin{array}{l}\text { No quantitative analysis was } \\
\text { provided. Qualitative description } \\
\text { of number of imitated sounds } \\
\text { and words at the end of intensive } \\
\text { training were included for all four } \\
\text { children: } \\
\text { Child } 1 \text { : Imitated all vowel and } \\
\text { consonant sounds, } 25 \text { words/ } \\
\text { word approximations } \\
\text { Child } 2 \text { : Almost all vowel and } \\
\text { consonant sounds, at least } 60 \\
\text { words/word approximations } \\
\text { Child } 3: \text { Most vowel and conso- } \\
\text { nant sounds, at least } 16 \text { words/ } \\
\text { word approximations } \\
\text { Child } 4 \text { : Imitated } 9 \text { sounds } \\
\text { Authors concluded that "intensive } \\
\text { training combined with elevated } \\
\text { motivational levels can lead to } \\
\text { substantial increases in the be- } \\
\text { havior of mute autistic children". }\end{array}$ \\
\hline $\begin{array}{l}\text { Jones EA } \\
{[17]}\end{array}$ & $\begin{array}{l}2(2,0) \\
3 ; 2 \& \\
4 ; 11\end{array}$ & $\begin{array}{l}\text { Joint Attention } \\
\text { Hybrid. Included } \\
\text { nonverbal and } \\
\text { coordinated with } \\
\text { verbal. }\end{array}$ & $\begin{array}{l}\text { Single case design on } \\
\text { several measures of } \\
\text { joint attention }\end{array}$ & $\begin{array}{l}\text { PRT for Gaze, Gaze } \\
\text { Alternating, Gaze Al- } \\
\text { ternating and Pointing, } \\
\text { and Gaze Alternating, } \\
\text { Pointing and Verbal- } \\
\text { ization }\end{array}$ & $\begin{array}{l}\text { Both children learned the target } \\
\text { behaviors } \\
\text { JA attention alone did not result } \\
\text { in improvements in pointing or } \\
\text { verbalizations; Pointing and ver- } \\
\text { balizations improved only after } \\
\text { they were specifically targeted }\end{array}$ \\
\hline $\begin{array}{l}\text { Kasari C, et } \\
\text { al. [12] }\end{array}$ & $\begin{array}{l}61(51 \\
10) \\
5-8\end{array}$ & $\begin{array}{l}\text { From } 20 \text { min } \\
\text { Natural language } \\
\text { sample Total \# } \\
\text { of spontaneous } \\
\text { communicative ut- } \\
\text { terance. Also \# dif- } \\
\text { ferent word roots } \\
\text { and \# of comments }\end{array}$ & $\begin{array}{l}\text { Pre post naturalistic } \\
\text { language samples }\end{array}$ & $\begin{array}{l}\text { JASPER-EMT alone } \\
\text { or JASPER-EMT+S- } \\
\text { GD. First } 3 \text { months } \\
\text { 2-hour long sessions } \\
\text { - increasing to } 3 \text { hours } \\
\text { per week for slow re- } \\
\text { sponders }\end{array}$ & $\begin{array}{l}\text { JASP + EMT + SGT greatest } \\
\text { gains }\end{array}$ \\
\hline $\begin{array}{l}\text { Koegel RL, } \\
\text { et al. [35] }\end{array}$ & $\begin{array}{l}2 \text { (Not } \\
\text { report- } \\
\text { ed) } \\
4 ; 5-5 ; 8\end{array}$ & $\begin{array}{l}\text { Number of utter- } \\
\text { ances, sponta- } \\
\text { neous-imitation }\end{array}$ & $\begin{array}{l}\text { Single subject design } \\
\text { No pre-post tests }\end{array}$ & NLP and Analog (DT) & $\begin{array}{l}\text { Improvements in the number of } \\
\text { imitated and spontaneous utter- } \\
\text { ances }\end{array}$ \\
\hline $\begin{array}{l}\text { Koegel RL, } \\
\text { et al. [18] }\end{array}$ & $\begin{array}{l}3(3,0) \\
3 ; 0-4 ; 8\end{array}$ & $\begin{array}{l}\text { CDI, \%Correct } \\
\text { Verbalizations }\end{array}$ & $\begin{array}{l}\text { Single subject design } \\
\text { No pre-post tests }\end{array}$ & $\begin{array}{l}\text { PRT (Antecedent } \\
\text { Stimulus Control: Us- } \\
\text { ing Orienting Cues) }\end{array}$ & $4,38 \& 245$ words $(n=3)$ \\
\hline $\begin{array}{l}\text { Koegel RL, } \\
\text { et al. [19] }\end{array}$ & $\begin{array}{l}3(3,0) \\
3 ; 2-3 ; 5\end{array}$ & $\begin{array}{l}\text { \# Reinforcer } \\
\text { strength } \\
\text { \# Self-initiated } \\
\text { social engagement } \\
\text { during communi- } \\
\text { cation } \\
\text { \# Nonverbal dyad- } \\
\text { ic orienting } \\
\text { \# General child } \\
\text { affect }\end{array}$ & $\begin{array}{l}\text { Single subject design } \\
\text { No pre-post tests }\end{array}$ & $\begin{array}{l}\text { Embedding social in- } \\
\text { teraction into child-pre- } \\
\text { ferred reinforcer in a } \\
\text { naturalistic language } \\
\text { intervention context }\end{array}$ & $\begin{array}{l}\text { \# Reinforcer strength } \\
\text { Comparable across both condi- } \\
\text { tions } \\
\text { during communication } \\
\text { Improvement in in self-initiated } \\
\text { social engagement, } \\
\text { nonverbal dyadic orienting and } \\
\text { affect in all three children. }\end{array}$ \\
\hline $\begin{array}{l}\text { Laski KE, } \\
\text { et al. [23] }\end{array}$ & $\begin{array}{l}8(7,1) \\
5-9.6\end{array}$ & $\begin{array}{l}\text { Parent verbaliza- } \\
\text { tions, child vocal- } \\
\text { izations (imitations, } \\
\text { answers, sponta- } \\
\text { neous speech) }\end{array}$ & $\begin{array}{l}\text { Parent verbalizations, } \\
\text { child vocalizations } \\
\text { (imitations, answers, } \\
\text { spontaneous speech) }\end{array}$ & $\begin{array}{l}\text { Natural language par- } \\
\text { adigm (NLP) with clinic } \\
\text { sessions and parent } \\
\text { implementation }\end{array}$ & $\begin{array}{l}\text { Parents increased the frequency } \\
\text { with which they required their } \\
\text { children to speak (i.e., modeled } \\
\text { words and phrases, prompted } \\
\text { answers to questions); all partic- } \\
\text { ipants with autism increased the } \\
\text { frequency of verbalizations in } 3 \\
\text { non-training settings }\end{array}$ \\
\hline
\end{tabular}




\begin{tabular}{|c|c|c|c|c|c|}
\hline $\begin{array}{l}\text { Miller A, et } \\
\text { al. [38] }\end{array}$ & $\begin{array}{l}19(12 \\
7) \\
5-23\end{array}$ & $\begin{array}{l}\text { Expressive and re- } \\
\text { ceptive words and } \\
\text { signs but not clear } \\
\text { how data were col- } \\
\text { lected. } \\
\text { Hybrid. Did include } \\
\text { spoken words }\end{array}$ & No pre-post tests & $\begin{array}{l}\text { Cognitive-develop- } \\
\text { mental; } \\
\text { Participants walked } \\
\text { on parallel boards 3-6' } \\
\text { above the ground with } \\
\text { obstacles to become } \\
\text { aware and decrease } \\
\text { "autistic mannerisms" } \\
\text { Next, } 50 \text { function- } \\
\text { al ASD signs were } \\
\text { taught via signs on the } \\
\text { boards, training films, } \\
\text { and generalization to } \\
\text { everyday contexts; } \\
\text { adults taught to elicit } \\
\text { signs throughout day } \\
\text { paired with spoken } \\
\text { word }\end{array}$ & $\begin{array}{l}\text { All improved in receptive and } \\
\text { expressive use of responding to } \\
\text { signs. Children respond to more } \\
\text { signs than used them. } 7 \text { of the } 19 \\
\text { children produced some spoken } \\
\text { words relating to the signs (range } \\
1-50 \text { with only one participant } \\
\text { above } 7 \text { ) } \\
\text { Duration of training correlated } \\
\text { with higher Creak scores } \\
\text { Day school students performed } \\
\text { better than residential. }\end{array}$ \\
\hline $\begin{array}{l}\text { Ozonoff S, } \\
\text { et al. [36] }\end{array}$ & $\begin{array}{l}22(18 \\
4) \\
2 ; 7-5 ; 9\end{array}$ & $\begin{array}{l}\text { The Psychoeduca- } \\
\text { tional Profile-Re- } \\
\text { vised. Hybrid: } \\
\text { Words are includ- } \\
\text { ed in the overall } \\
\text { PEP-R score, but } \\
\text { actual word use/ } \\
\text { vocalizations were } \\
\text { not included. }\end{array}$ & $\begin{array}{l}\text { The Psychoeducation- } \\
\text { al Profile-Revised sub- } \\
\text { scales: Imitation, Per- } \\
\text { ception, Fine Motor, } \\
\text { Gross Motor, Hand- } \\
\text { eye Integration, Cog- } \\
\text { nitive-Performance, } \\
\text { Cognitive-Verbal and } \\
\text { Total PEP-R score }\end{array}$ & $\begin{array}{l}\text { Parent Implemented } \\
\text { TEACCH or Control } \\
\text { ( } n=11 \text { in each group) }\end{array}$ & $\begin{array}{l}\text { TEACCH Group bigger gains } \\
\text { than control group on Imitation, } \\
\text { Fine Motor, Gross Motor, Cog- } \\
\text { nitive-Performance, and Total } \\
\text { PEP-R score }\end{array}$ \\
\hline $\begin{array}{l}\text { Oxman J, } \\
\text { et al. [24] }\end{array}$ & $\begin{array}{l}10(5,5) \\
9 ; 1-9 ; 5\end{array}$ & $\begin{array}{l}\text { Speech produc- } \\
\text { tion/imitation cate- } \\
\text { gorized by: } \\
\text { (1) Willingness/ } \\
\text { motivation to vo- } \\
\text { calize } \\
\text { (2) Participants' } \\
\text { precise speech } \\
\text { abilities }\end{array}$ & $\begin{array}{l}\text { Fisher-Logemann Test } \\
\text { of Articulation Compe- } \\
\text { tence } \\
\text { Test consists of } 106 \\
\text { items - three trials giv- } \\
\text { en for each test item } \\
\text { speech pathologists } \\
\text { used two measures } \\
\text { to score responses: } \\
\text { First trial of each test } \\
\text { item was scored for } \\
\text { presence/absence of } \\
\text { a vocal response, re- } \\
\text { gardless of its quality } \\
\text { (willingness/motivation } \\
\text { to vocalize); vocal re- } \\
\text { sponses were scored } \\
\text { in terms of their articu- } \\
\text { latory correctness (or } \\
\text { correspondence with } \\
\text { the examiner's speech } \\
\text { models) (used to mea- } \\
\text { sure participants' pre- } \\
\text { cise speech abilities) }\end{array}$ & $\begin{array}{l}\text { Simultaneous com- } \\
\text { munication training } \\
\text { (speaking and signing) } \\
\text { compared with a con- } \\
\text { trol group that received } \\
\text { vocal responding } \\
\text { (speech-oriented so- } \\
\text { cialization program) }\end{array}$ & $\begin{array}{l}\text { Simultaneous communication } \\
\text { did not result in improvements in } \\
\text { any of the nonverbal participants, } \\
\text { however control group } \\
\text { Significant increase in the ex- } \\
\text { perimental group's pre to post- } \\
\text { test performance on first trial } \\
\text { responses; no improvement in } \\
\text { the control group; all children per- } \\
\text { formed at very low levels for the } \\
\text { measure of articulatory correct- } \\
\text { ness at both pre/post-testing - no } \\
\text { significant differences between } \\
\text { the pre- and post-test scores }\end{array}$ \\
\hline
\end{tabular}




\begin{tabular}{|c|c|c|c|c|c|}
\hline $\begin{array}{l}\text { Rogers SJ, } \\
\text { et al. [37] }\end{array}$ & $\begin{array}{l}10(10, \\
0) \\
1 ; 8-5 ; 5\end{array}$ & $\begin{array}{l}\text { \# Novel words or } \\
\text { approximations; } \\
\text { \# novel phrases } \\
\text { (also looked at } \\
\text { function of com- } \\
\text { munication, and } \\
\text { if utterance was } \\
\text { prompted or spont; } \\
\text { Frequency of } \\
\text { speech }\end{array}$ & $\begin{array}{l}\text { ADOS, SCQ, Mullen, } \\
\text { VABS, CDI, } 15 \text { min } \\
\text { speech probes } \\
1 \text { press for request } \\
\text { and } 1 \text { for JA ("look") } \\
\text { FU - speech probe af- } \\
\text { ter } 3 \text { months }\end{array}$ & $\begin{array}{l}\text { Denver Model (behav- } \\
\text { ioral, developmental, } \\
\text { and relationship-ori- } \\
\text { ented intervention) or } \\
\text { PROMPT (neuro-de- } \\
\text { velopmental approach } \\
\text { for speech production } \\
\text { disorders) } \\
1 \text { hour per week for } 12 \\
\text { hours }\end{array}$ & $\begin{array}{l}\text { 8/10 children demonstrated func- } \\
\text { tional spontaneous use of } 5 \text { or } \\
\text { more novel words during therapy } \\
\text { and generalization (less during } \\
\text { play/gen) } \\
9 / 10 \text { improved on CDI } \\
\text { Higher Developmental quotient } \\
\text { did better } \\
\text { Age, cognitive abilities, imitation, } \\
\text { intentional skills and milder au- } \\
\text { tism symptoms may have moder- } \\
\text { ated success } \\
\text { Poorest outcomes: Attention, tol- } \\
\text { erating demands, participating, ja } \\
\text { Good: Mild autism, social orient- } \\
\text { ing }\end{array}$ \\
\hline $\begin{array}{l}\text { Sandiford } \\
\text { GA, et al. } \\
{[25]}\end{array}$ & $\begin{array}{l}12 \\
(11 / 1) \\
5 ; 0-7 ; 6\end{array}$ & $\begin{array}{l}\text { \#Verbal Attempts } \\
\text { (Correct Words) } \\
\text { \#Words Parent } \\
\text { Report } \\
\text { \# Imitative At- } \\
\text { tempts }\end{array}$ & $\begin{array}{l}\text { Number of verbal at- } \\
\text { tempts, number of cor- } \\
\text { rect words, number of } \\
\text { words reported by the } \\
\text { parent, and number of } \\
\text { imitative attempts. In } \\
\text { order to measure num- } \\
\text { ber of verbal attempts } \\
\text { and number of } \\
\text { correct words over } \\
\text { time, a } \\
\text { criterion referenced } \\
\text { vocabulary } \\
\text { test developed by the } \\
\text { first author was given } \\
\text { at baseline and the be- } \\
\text { ginning of each treat- } \\
\text { ment week. }\end{array}$ & $\begin{array}{l}\text { Melodic Intonation Tx } \\
n=5 \\
\text { Standard Tx } n=5\end{array}$ & $\begin{array}{l}\text { Pre-post gains in \#VACR, PR \& } \\
\text { IA for MIT } \\
\text { Pre-post gains in \#VACR, } \\
\text { No diff between groups on } \\
\text { \#VACR, \#PR or \#IA }\end{array}$ \\
\hline $\begin{array}{l}\text { Scanlan } \\
\text { JB, et al. } \\
\text { [26] }\end{array}$ & $\begin{array}{l}8(7,1) \\
5 ; 2-9 ; 6\end{array}$ & None reported & No pre-post measures & $\begin{array}{l}\text { Treatment empha- } \\
\text { sized activities that } \\
\text { encouraged interaction } \\
\text { between the therapist } \\
\text { and the child (e.g., us- } \\
\text { ing pictures of familiar } \\
\text { objects, animals and } \\
\text { people). Therapist la- } \\
\text { beled each picture and } \\
\text { encouraged the child } \\
\text { to look at the thera- } \\
\text { pist's mouth. A hearing } \\
\text { tube was occasionally } \\
\text { used to stimulate ver- } \\
\text { bal communication. } \\
\text { Child's lips and jaw } \\
\text { were manually manip- } \\
\text { ulated sometimes to } \\
\text { stimulate speech. }\end{array}$ & $\begin{array}{l}\text { Qualitative description: "gains in } \\
\text { verbal expression, however, did } \\
\text { not nearly approach the gains } \\
\text { made in verbal comprehension" }\end{array}$ \\
\hline
\end{tabular}




\begin{tabular}{|c|c|c|c|c|c|}
\hline $\begin{array}{l}\text { Schreib- } \\
\text { man L, et } \\
\text { al. [20] }\end{array}$ & $\begin{array}{l}39(34, \\
5) \\
1 ; 6-3 ; 75\end{array}$ & $\begin{array}{l}\text { CDI Mullen Vine- } \\
\text { land }\end{array}$ & $\begin{array}{l}\text { The Mullen Scales of } \\
\text { Early } \\
\text { Learning (MSEL), Ex- } \\
\text { pressive } \\
\text { One-Word Picture Vo- } \\
\text { cabulary } \\
\text { Test-Revised (EOW- } \\
\text { PVT), } \\
\text { MacArthur Communi- } \\
\text { cative } \\
\text { Developmental Inven- } \\
\text { tory (CDI), } \\
\text { Vineland Adaptive Be- } \\
\text { havior } \\
\text { Scales (VABS), PECS } \\
\text { use level } \\
\text { score (1 - 6), Parent } \\
\text { Satisfaction } \\
\text { Survey }\end{array}$ & $\begin{array}{l}\text { PRT } n=20 \\
\text { PECS } n=19\end{array}$ & No difference \\
\hline $\begin{array}{l}\text { Shire SY, } \\
\text { et al. [13] } \\
\text { "Partial } \\
\text { data from } \\
1 \text { site of a } \\
\text { multi-site } \\
\text { study }\end{array}$ & $\begin{array}{l}22(22, \\
0) \\
5-8\end{array}$ & $\begin{array}{l}\text { CCX 10-minute } \\
\text { parent interaction } \\
\text { - spontaneous } \\
\text { language and } \\
\text { function (request, } \\
\text { comment, other } \\
\text { non-social) }\end{array}$ & $\begin{array}{l}\text { 10-minute language } \\
\text { samples collected } \\
\text { monthly and at fol- } \\
\text { low-up - standard toys } \\
\text { parents asked to inter- } \\
\text { act as usual } \\
\text { No other measures }\end{array}$ & $\begin{array}{l}\text { JASPER-EMT alone or } \\
\text { JASPER-EMT + SGD }\end{array}$ & $\begin{array}{l}\text { Sig increase in spontaneous re- } \\
\text { quests \& comments } \\
\text { No sig difference bet time and } \\
\text { treatment condition } \\
\text { Comments "modestly significant" }\end{array}$ \\
\hline $\begin{array}{l}\text { Strasberger } \\
\text { SK, et al. } \\
{[27]}\end{array}$ & $\begin{array}{l}4(4,0) \\
5 ; 8- \\
12 ; 11\end{array}$ & $\begin{array}{l}\text { Frequency of in- } \\
\text { dependent 2-step } \\
\text { mand sentence } \\
\text { sequence; fre- } \\
\text { quency of respons- } \\
\text { es; generalization } \\
\text { measures in class- } \\
\text { room; teachers' } \\
\text { ratings on the Be- } \\
\text { havioral Interven- } \\
\text { tion Rating Scale } \\
\text { (BIRS); social va- } \\
\text { lidity measure for } \\
\text { peers }\end{array}$ & $\begin{array}{l}\text { Frequency of indepen- } \\
\text { dent 2-step mand sen- } \\
\text { tence sequence; fre- } \\
\text { quency of responses }\end{array}$ & $\begin{array}{l}\text { peer assisted commu- } \\
\text { nication application } \\
\text { (PACA) on iPod SGD }\end{array}$ & $\begin{array}{l}\text { All } 4 \text { participants were able to } \\
\text { use an iPod-based SGD for } \\
\text { some communicative purpose; } \\
2 \text { participants generalized and } \\
\text { maintained their new communi- } \\
\text { cation; classroom teachers and } \\
\text { same-aged peers reported the } \\
\text { intervention as being both ac- } \\
\text { ceptable and effective }\end{array}$ \\
\hline $\begin{array}{l}\text { Wan CY, et } \\
\text { al. [28] }\end{array}$ & $\begin{array}{l}6(5,1) \\
5 ; 9-8 ; 9\end{array}$ & $\begin{array}{l}\text { \% CV approxima- } \\
\text { tions }\end{array}$ & $\begin{array}{l}\text { Single subject design } \\
\text { No pre-post tests }\end{array}$ & $\begin{array}{l}\text { Auditory Motor; Map- } \\
\text { ping Training }\end{array}$ & $\begin{array}{l}\text { After therapy, all children showed } \\
\text { significant improvements in their } \\
\text { ability to articulate words; All six } \\
\text { subjects increased \%CV approx- } \\
\text { imations }\end{array}$ \\
\hline
\end{tabular}




\begin{tabular}{|c|c|c|c|c|c|}
\hline $\begin{array}{l}\text { Wetherby } \\
\text { AM, et al. } \\
{[21]}\end{array}$ & $\begin{array}{l}82(71, \\
11) \\
1 ; 4-1 ; 8\end{array}$ & $\begin{array}{l}\text { Social communi- } \\
\text { cation, } \\
\text { autism symptoms, } \\
\text { adaptive behavior, } \\
\text { and developmental } \\
\text { level }\end{array}$ & $\begin{array}{l}\text { Social, Speech, and } \\
\text { Symbolic composites } \\
\text { of the CSBS Behavior } \\
\text { Sample; Social Affect } \\
\text { (SA) and Repetitive } \\
\text { Behavior (RRB) } \\
\text { domains of ADOS; } \\
\text { Communication, Daily } \\
\text { Living, Socialization, } \\
\text { and Motor scores from } \\
\text { the Vineland Adap- } \\
\text { tive Behavior Scales } \\
\text { (VABS-II); Visual Re- } \\
\text { ception, Fine Motor, } \\
\text { Receptive Language, } \\
\text { and Expressive Lan- } \\
\text { guage scores from } \\
\text { The Mullen Scales of } \\
\text { Early Learning (MSEL) }\end{array}$ & $\begin{array}{l}\text { Comparing two inter- } \\
\text { ventions within the } \\
\text { Early Social Interaction } \\
\text { (ESI) Project: Individu- } \\
\text { al-ESI vs. group-ESI }\end{array}$ & $\begin{array}{l}\text { Participants in individual ESI } \\
\text { showed significantly greater } \\
\text { improvement on the Social com- } \\
\text { posite of CBSB; participants in } \\
\text { both groups showed significant } \\
\text { improvement in social affect and } \\
\text { worsening in RRBs of ADOS; } \\
\text { Participants in individual-ESI } \\
\text { showed significant improvement } \\
\text { in Communication and Daily Life } \\
\text { and stability in Socialization on } \\
\text { VABS; participants in group-ESI } \\
\text { showed no change in Communi- } \\
\text { cation and Daily Life and signif- } \\
\text { icant decrease in Socialization; } \\
\text { participants showed no signifi- } \\
\text { cant change in Visual Reception } \\
\text { of MSEL; participants in the } \\
\text { individual-ESI showed significant } \\
\text { improvement in Receptive Lan- } \\
\text { guage but there was no change } \\
\text { for participants in group-ESI }\end{array}$ \\
\hline $\begin{array}{l}\text { Yoder PJ, } \\
\text { et al. [29] }\end{array}$ & $\begin{array}{l}60 \text { (Not } \\
\text { report- } \\
\text { ed) } \\
\\
\text { Range } \\
\text { not re- } \\
\text { ported; } \\
\text { Mean } \\
5-5 ; 6 . \\
\text { SD } \\
1.2-2.1 \\
\text { across } \\
\text { groups }\end{array}$ & $\begin{array}{l}\text { Total number of } \\
\text { different child-initi- } \\
\text { ated spoken words } \\
\text { observed during } \\
40-\text { minute training } \\
\text { sessions }\end{array}$ & $\begin{array}{l}\text { Direct observation by } \\
\text { language clinician } \\
\text {-Recorded utterances } \\
\text { as they occurred } \\
\text {-Pre-treatment: Verbal } \\
\text { imitation was trained } \\
\text { and assessed using } \\
\text { the sum of correct } \\
\text { trials per item (lan- } \\
\text { guage clinicians asked } \\
\text { participants to verbally } \\
\text { imitate } 11 \text { items) }\end{array}$ & $\begin{array}{l}\text { Simultaneous presen- } \\
\text { tation of speech and } \\
\text { signing ( } 4 \text { groups: } \\
\text { Sign Alone, Speech } \\
\text { Alone, Simultaneous } \\
\text { presentation of Sign } \\
\text { and Speech, and Alter- } \\
\text { nating Presentation of } \\
\text { Sign and Speech) }\end{array}$ & $\begin{array}{l}\text { Sign Alone group used signifi- } \\
\text { cantly fewer spontaneous words } \\
\text { than participants in the other } 3 \\
\text { groups; Participants in the other } \\
3 \text { groups did not significantly dif- } \\
\text { fer from each other; pretreatment } \\
\text { verbal imitation abilities (higher) } \\
\text { were predictive of spontaneous } \\
\text { oral language use by } 57 \text { of the } \\
60 \text { participants }\end{array}$ \\
\hline
\end{tabular}

checked articles for accuracy. Numerical findings in the Results were checked, and five that were below $100 \%$ were analyzed by another author independently and the matching score was reported.

\section{Results}

\section{Assessment}

Four studies used the same data base (same participants), with similar findings, therefore only one study with the largest $\mathrm{N}$ was used in the calculations [10-13]. There were 649 unique participants with ASD that received intervention in the 26 independent studies. Twenty-four of the studies reported number of males and females included; $84 \%$ of participants were males $(n=496)$ and $16 \%$ of participants were females $(n=91)$. Gender was not reported for 62 participants. The age span of the participants ranged from 1 year 4 months to 23 years. A closer analysis revealed that eight studies exclusively targeted children in the toddler/preschool years (under 4 years 11 months), with a total of 295 participants [14-21]. Eight studies included participants exclusively in elementary school (ages 5-12;11) with 115 participants [10,22-33]. Participants in nine studies [6,31-37] included a combination of preschool and elementary school aged children with 220 participants. The re- maining study included a combination of elementary/adolescents/adults with 19 participants [38]. In regard to the assessment of change measures, most studies (77\%) had direct measures of speech sounds, word approximations, words, or utterances. Two studies only reported standardized test scores (PEP-R, CDI/Mullen/Vineland) [20,36]. One study measured behaviors related to communication (social engagement/affect) and described each participant's outcome [19].

\section{Treatment}

Most studies reported improvements in communication. In regard to interventions for toddler and preschool children, seven of the eight studies used strategies based on Applied Behavior Analysis (ABA); two used traditional $A B A$ discrete trials procedures such as modeling, shaping, and rewarding desired behavior $[14,16]$, and three studies used Pivotal Response Treatment (PRT) that focuses on motivational components (e.g., child choice, natural rewards, task variation, rewarding attempts) $[17,18,20]$. One study used a parent-mediated intervention that focused on parent responsiveness to child communication and using communication during action routines [15]. The remaining study targeted engagement in natural 
environments, social communication, and emotional regulation [21]. One study compared a picture system (PECS) to a verbal only intervention (PRT) and found no group differences [20]. Another study compared group parent education with individual parent education and found that, while all children improved, the individualized parent education group demonstrated greater improvements in communicative areas [21].

For elementary school-aged children seven of the twelve studies added some type of augmentative device, such as pictures, computer, gestures, or signs in addition to verbal communication. Five studies focused on verbal communication without an augmentative system. Of those five studies that used various verbal approaches (pre-linguistic milieu Teaching [22], NLP/PRT [35], Melodic Intonation Treatment (MIT) [25], a non-labeled intervention that focused on modeling/attention/ hearing tube/manual manipulation, and auditory motor mapping [37]) all resulted in improvements in verbal communication. However, MIT participants did not show significantly greater outcomes than those receiving a traditional treatment. In regard to interventions that used a combination of treatments, one study found that a combination of a speech generating device (SGD) and verbal production was more effective than an intervention focusing on joint attention, play, engagement $\&$ regulation (JASPER), with a verbal component [10].

Another study that used a picture system of communication (PECS) found that some children improved in their use of requests (both verbal and with the picture cards), but PECS did not result in improvements in communication used for social purposes [33]. Further, the picture system did not enhance the verbal communication of the children who were already using some verbal communication at baseline. A study using simultaneous communication (speaking and signing together) compared with a verbal only approach found no difference between the verbal outcomes of the groups [24]. Finally, a study that compared sign alone, speech alone, sign with speech, and alternating sign and speech found that the only group that used significantly fewer spontaneous words was the sign only group [29].

Studies that included a combination of preschoolers and elementary school aged children did not use augmentative communication but, similar to the preschool studies, directly targeted communication. One used Auditory Motor Mapping Training alone and compared this with added Speech Repetition Therapy. The AMMT + SPT produced the best outcomes [30]. Several studies used ABA or naturalistic ABA procedures and those studies showed improvements in verbal communication (varied vocalizations, vocalizations, words) in all participants [22,34,36,37]. One study used the Treatment and Education of Autistic and Communication related handicapped Children program (TEACCH) but did not report communicative gains [36] and another compared the Denver Model to Prompts for Restructuring Oral Muscular Phonetic Targets (PROMPT) techniques and showed greater gains with the Denver model [37].

Finally, the study that included a large age range (i.e., elementary/adolescents/adults) primarily included nonverbal participants and began with having the participants walk on uneven parallel bars above the ground then added pictures with signs and verbal modeling of the word [38]. After intervention researchers found improvements in sign use for all participants but only 7/19 participants learned some spoken words.

Moreover, this systematic review suggested that a majority of the participants in the studies, aged between $1 ; 4$ to 23 years, demonstrated communicative gains following intervention. Several studies suggested moderating factors. For example, children with higher skills at the start of intervention had a more positive response to the intervention. Measured pretreatment areas that were suggestive of more positive communicative outcomes included greater sound imitation, more mild symptoms of ASD, and the presence of social orienting. Additionally, the presence of functional language by age five was reported to be correlated with more positive outcomes in children with ASD. Overall, the level of evidence from these studies must be classified as preliminary or weak and the overall data available is quite limited, especially relative to that for verbal children with ASD.

\section{Discussion}

Nonverbal and minimally verbal individuals with ASD arguably represent the phenotype with the greatest support needs; however, this subgroup has been largely understudied in the research literature. Our searches through nearly 60 years of research only yielded 29 studies (and only 26 with unique participants) that focused exclusively on verbal communication to nonverbal or minimally verbal individuals with ASD. Within this literature base, there was a variety of reasons that precluded us from making conclusive evidence-based recommendations and made it difficult to compare findings across studies. These included: (1) Definitions: A lack of uniform terminology for defining "nonverbal" and "minimally verbal" was noted across studies. Some classified individuals who could say a few words as "nonverbal" and other studies combined nonverbal and minimally verbal children in their research. Further, there was great heterogeneity in age groups with some studies focusing exclusively on preschool children (who could also be considered preverbal or prelinguistic) while other studies included elementary school aged children, adolescents, and adults. As such, the most effective interventions based on verbal status and age could not be determined due to a small number of studies in each area; (2) Measurement Systems: The reviewed 
studies varied greatly in how pretreatment behaviors were measured, how progress and outcomes were measured, and whether assessments included various settings and different communicative partners. The field requires a more uniform paradigm; standardized measures across settings would allow for more precise comparisons of procedures and systematic aggregation of findings across studies. Additionally, a greater understanding of prognostic pretreatment indicators (particularly in preschoolers) would be helpful in understanding the relative communicative gains of an intervention, moderating factors, and the likelihood of response to intervention; (3) Diversity of Interventions: Although the most common effective procedures were rooted in applied behavior analysis principles ( $A B A)$, there was great variability in intervention procedures within $A B A$ (i.e., discrete trials, pivotal response training) and for non-ABA procedures (auditory-visual mapping, Melodic Intonation Therapy), again creating challenges in understanding best practices. Given these challenges, we were still able to make some general preliminary findings, as follow.

Grouping non- and minimally verbal children across age groups did result in some interesting patterns in the research within the limitations described previously: (1) A verbal only approach is recommended for toddlers and preschoolers. All studies showed improvements in verbal communication using a speech only approach for preschoolers. One study comparing an augmentative vs. a speech only approach did not show that a picture system resulted in greater improvements. One question in for nonverbal children with ASD has been whether the use of an augmentative system would facilitate expressive verbal communication. This review did not indicate this combination was differentially beneficial. (2) Research suggests that targeting pre-linguistic behaviors may not lead to improvements in verbal communication [17]. The most successful programs appeared to directly target verbal communication. Further research on learning trajectories and prelinguistic behaviors should help elucidate those who will become verbal and those prelinguistic areas that potentially could be taught to accomplish better outcomes for all children. (3) When children reach elementary school age, while a greater diversity in treatments was noted, more than half of the studies showed gains in communication using various communicative interventions without an augmentative device. Only one set of participants $[10,12,13]$ (the

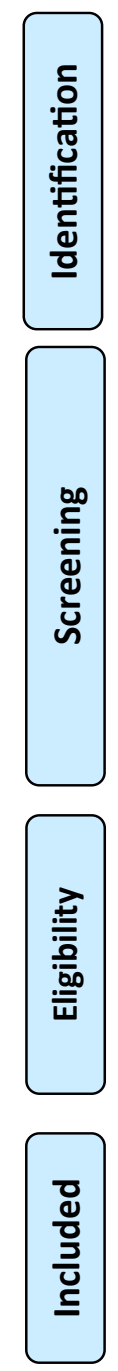

$$
\begin{aligned}
& \text { Records identified through database } \\
& \text { searching } \\
& (\mathrm{n}=2007)
\end{aligned}
$$

Additional records identified through other sources

$$
(\mathrm{n}=1)
$$

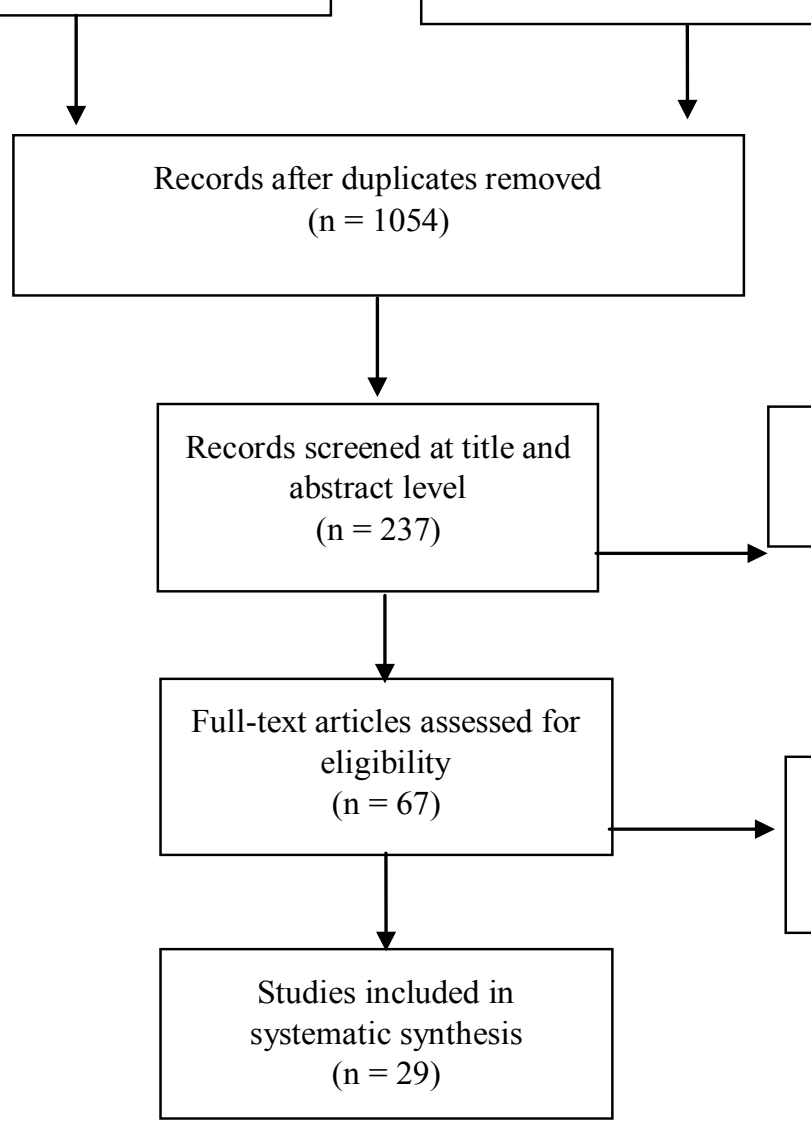

Full-text articles excluded, with reasons $(\mathrm{n}=170)$

Figure 1: PRISMA 2009 flow diagram [39]. 
same participants reported in four published studies) found that a combination of a speech generating device was more effective than intervention alone, however, their intervention was not solely focused on communication. As a whole, these results suggest that treatment focused on non-communication areas (e.g., play, joint attention, attentiveness) in combination with targeting verbal communication may not produce as large of gains in expressive communication as interventions that focus exclusively and directly on verbal communication.

More research is warranted in this area. (4) It does not appear necessary or helpful to manually manipulate the articulators during intervention for children with ASD (e.g., PROMPT). (5) Naturalistic interventions based on ABA procedures, providing considerable opportunities in everyday settings with a parent education component are the most well researched and currently appear to be most effective at producing expressive communication in preschool and elementary school aged children. (6) As a whole, the studies indicated that children who enter intervention at a higher level will also exit intervention at a higher level. Nonverbal children with higher levels of behaviors such as play, joint attention, sound imitation, gross motor imitation, attentiveness, socialization, along with less severe autism symptomology will begin talking sooner. Those with significant deficits in these areas are more likely to remain nonverbal.

In summary, this systematic review indicates the general paucity of intervention studies on minimally verbal or nonverbal children with ASD despite the long-standing high priority for developing effective treatments for these children (e.g., Interagency Council on Autism, 2011, 2017) [3]. The current lack of systematicity reduces available evidence-based treatment options. When comparing the definitions of the studies reviewed, one has to bear in mind that the considerable differences between the studies evaluated makes is difficult to extrapolate the existing studies to practice and to aggregate studies for meta analyses. Furthermore, studies using the same subject pools adversely affect the external validity of an intervention. Research should explicitly state whether the studies involve unique participants.

Despite these challenges, the current literature is a foundation to build future studies and systematic improvements are needed. Moreover, our attempt to accurately describe the current literature in regard to intervention recommendations for nonverbal and minimally verbal participants was hindered by the heterogeneity in the symptoms, varying ages, and different treatments and core lack of systematic, replicated intervention research paradigms that made it difficult to make definitive recommendations for intervention. We do acknowledge the possibility that some studies were missed in the analysis due to non-inclusion of the key words used but do point to the breadth of the original article catchment as reducing the likelihood that a large number of studies were missed. Future research is crucial for addressing the communication needs of the most severely impacted and underserved children with ASD (Figure 1) [39].

\section{Acknowledgments}

The authors wish to express gratitude to the Kind World Foundation, the Weitz Family Foundation, and the Scottish Rite Foundation of Nashville, Tennessee, USA for financial support of this review.

\section{References}

1. De Giacomo A, Fombonne E (1998) Parental recognition of developmental abnormalities in autism. Eur Child Adolesc Psychiatry 7: 131-136.

2. Franchini M, Duku E, Armstrong V, Brian J, Bryson SE, et al. (2018) Variability in verbal and nonverbal communication in infants at risk for autism spectrum disorder: Predictors and outcomes. J Autism Dev Disord 48: 3417-3431.

3. Interagency Autism Coordinating Committee (2017) IACC Strategic Plan for Autism Spectrum Disorder 2016-2017 update.

4. Tager-Flusberg H, Kasari C (2013) Minimally verbal schoolaged children with autism spectrum disorder: The neglected end of the spectrum. Autism Res 6: 468-478.

5. Stedman A, Taylor B, Erard M, Peura C, Siegel M (2019) Are children severely affected by autism spectrum disorder underrepresented in treatment studies? An analysis of the literature. J Autism Dev Disord 49: 1378-1390.

6. Chenausky B (2017) Commentary: Critical considerations for studying low-functioning autism. J Child Psychol Psychiatry 58: 436-438.

7. American Psychiatric Association (2013) Diagnostic and statistical manual of mental disorders. ( $5^{\text {th }}$ edn), Arlington, USA.

8. Howlin P (2003) Can early interventions alter the course of autism? Novartis Found Symp 250-265.

9. Rose V, Trembath D, Keen D, Paynter J (2016) The proportion of minimally verbal children with autism spectrum disorder in a community-based early intervention programme. J Intellect Disabil Res 60: 464-477.

10. Almirall D, DiStefano C, Chang YC, Shire S, Kaiser A, et al. (2016) Longitudinal effects of adaptive interventions with a speech-generating device in minimally verbal children with ASD. J Clin Child Adolesc Psychol 45: 442-456.

11. DiStefano C, Shih W, Kaiser A, Landa R, Kasari C (2016) Communication growth in minimally verbal children with ASD: The importance of interaction. Autism Res 9: 10931102.

12. Kasari C, Kaiser A, Goods K, Nietfeld J, Mathy P, et al. (2014) Communication interventions for minimally verbal children with autism: A sequential multiple assignment randomized trial. J Am Acad Child Adolesc Psychiatry 53: 635-646.

13. Shire SY, Goods K, Shih W, DiStefano C, Kaiser A, et al. (2015) Parents' adoption of social communication intervention strategies: Families including children with autism spectrum disorder who are minimally verbal. J Autism Dev Disord 45: 1712-1724. 
14. Drash PW, High RL, Tudor RM (1999) Using mand training to establish an echoic repertoire in young children with autism. Anal Verbal Behav 16: 29-44.

15. Green J, Charman T, McConachie H, Aldred C, Slonims $V$, et al. (2010) Parent-mediated communication-focused treatment in children with autism (PACT): A randomised controlled trial. Lancet 375: 2152-2160.

16. Harris SL, Wolchik SA, Milch RE (1983) Changing the speech of autistic children and their parents. Child \& Family Behavior Therapy 4: 151-173.

17. Jones EA (2009) Establishing response and stimulus classes for initiating joint attention in children with autism. Research in Autism Spectrum Disorders 3: 375-389.

18. Koegel RL, Shirotova L, Koegel LK (2009) Brief report: Using individualized orienting cues to facilitate first-word acquisition in non-responders with autism. J Autism Dev Disord 39: 1587-1592.

19. Koegel RL, Vernon TW, Koegel LK (2009) Improving social initiations in young children with autism using reinforcers with embedded social interactions. J Autism Dev Disord 39: 1240-1251.

20. Schreibman L, Stahmer AC (2014) A randomized trial comparison of the effects of verbal and pictorial naturalistic communication strategies on spoken language for young children with autism. J Autism Dev Disord 44: 1244-1251.

21. Wetherby AM, Guthrie W, Woods J, Schatschneider C, Holland RD, et al. (2014) Parent-implemented social intervention for toddlers with autism: An RCT. Pediatrics 134: 1084-1093.

22. Franco JH, Davis BL, Davis JL (2013) Increasing social interaction using prelinguistic milieu teaching with nonverbal school-age children with autism. Am J Speech Lang Pathol 22: 489-502.

23. Laski KE, Charlop MH, Schreibman L (1988) Training parents to use the natural language paradigm to increase their autistic children's speech. J Appl Behav Anal 21: 391 400.

24. Oxman J, Konstantareas MM, Liebovitz-Bojm SF (1979) Simultaneous communication training and vocal responding in nonverbal autistic and autistic-like children. Int J Rehabil Res 2: 394-395.

25. Sandiford GA, Mainess KJ, Daher NS (2013) A pilot study on the efficacy of melodic based communication therapy for eliciting speech in nonverbal children with autism. J Autism Dev Disord 43: 1298-1307.

26. Scanlan JB, Leberfeld D, Freibrun R (1963) Language training in the treatment of the autistic child functioning on a retarded level. Mental retardation 1: 305-310.
27. Strasberger SK, Ferreri SJ (2014) The effects of peer assisted communication application training on the communicative and social behaviors of children with autism. J Dev Phys Disabil 26: 513-526.

28. Wan CY, Bazen L, Baars R, Libenson A, Zipse L, et al. (2011) Auditory-motor mapping training as an intervention to facilitate speech output in non-verbal children with autism: A proof of concept study. PLoS One 6: e25505.

29. Yoder PJ, Layton TL (1988) Speech following sign language training in autistic children with minimal verbal language. $J$ Autism Dev Disord 18: 217-229.

30. Chenausky K, Norton A, Tager-Flusberg H, Schlaug G (2016) Auditory-motor mapping training: Comparing the effects of a novel speech treatment to a control treatment for minimally verbal children with autism. PLoS One 11: e0164930.

31. Esch JW, Esch BE, Love JR (2009) Increasing vocal variability in children with autism using a lag schedule of reinforcement. Anal Verbal Behav 25: 73-78.

32. Gevarter C, Horan K (2018) A behavioral intervention package to increase vocalizations of individuals with autism during speech-generating device intervention. Journal of Behavioral Education 1-27.

33. Gordon K, Pasco G, McElduff F, Wade A, Howlin P, et al. (2011) A communication-based intervention for nonverbal children with autism: What changes? Who benefits? J Consult Clin Psychol 79: 447-457.

34. Hingtgen JN, Churchill DW (1969) Identification of perceptual limitations in mute autistic children: Identification by the use of behavior modification. Arch Gen Psychiatry 21: 68-71.

35. Koegel RL, O'Dell MC, Koegel LK (1987) A natural language teaching paradigm for nonverbal autistic children. J Autism Dev Disord 17: 187-200.

36. Ozonoff S, Cathcart K (1998) Effectiveness of a home program intervention for young children with autism. J Autism Dev Disord 28: 25-32.

37. Rogers SJ, Hayden D, Hepburn S, Charlifue-Smith R, Hall $\mathrm{T}$, et al. (2006) Teaching young nonverbal children with autism useful speech: A pilot study of the Denver model and PROMPT interventions. J Autism Dev Disord 36: 10071024.

38. Miller A, Miller EE (1973) Cognitive-developmental training with elevated boards and sign language $\mathrm{J}$ Autism Child Schizophr 3: 65-85.

39. Moher D, Liberati A, Tetzlaff J, Altman DG, The PRISMA Group (2009) Preferred reporting items for systematic reviews and meta-analyses: The PRISMA Statement. PLoS Med 6: e1000097. 